\title{
A polarimetric study of asteroids: fitting phase-polarization curves
}

\author{
A. Cellino, ${ }^{1 \star}$ S. Bagnulo, ${ }^{2}$ R. Gil-Hutton, ${ }^{3 \star}$ P. Tanga,${ }^{4}$ M. Canada-Assandri ${ }^{3}$ \\ and E. F. Tedesco ${ }^{5 \star}$ \\ ${ }^{1}$ INAF - Osservatorio Astrofisico di Torino, I-10025 Pino Torinese, Italy \\ ${ }^{2}$ Armagh Observatory, College Hill, Armagh BT61 9DG, UK \\ ${ }^{3}$ CASLEO and San Juan National University, J5402DSP, San Juan, Argentina \\ ${ }^{4}$ Observatoire de la Côte d'Azur, F-06304 Nice Cedex 4, France \\ ${ }^{5}$ Planetary Science Institute, Tucson, AZ 85719-2395, USA
}

Accepted 2015 October 20. Received 2015 October 19; in original form 2015 May 7

\begin{abstract}
By considering all published asteroid linear polarization data available in the literature, it is possible to obtain updated phase-polarization curves for several tens of objects. In a separate paper, we have produced new calibrations of different relations between the geometric albedo and several polarimetric parameters, based on an analysis of a limited sample of asteroids for which the albedo is known with sufficient accuracy. In this paper, we present the main polarization parameters and corresponding albedos for a larger data set of asteroids which we did not use for calibration purposes. We find a good agreement between the albedo values computed using different polarization parameters. Conversely, in the case of the so-called Barbarian asteroids the situation is rather unclear. Moreover, we present an updated analysis of the distributions of different polarimetric parameters, including the so-called inversion angle and the solar phase angle corresponding to the extreme value of negative polarization, and study their mutual relations. We find that the above parameters can be used to clearly distinguish some unusual classes of asteroids. Polarimetric parameters are known to be related to physical properties of asteroid surfaces which are difficult to infer by means of other observing techniques. By using a much larger data set, in our analysis we confirm and extend some results obtained in the past by other authors, and we explore more systematically some features that had been mostly unexplored before, mainly concerning the morphology of the negative polarization branch.
\end{abstract}

Key words: polarization-minor planets, asteroids: general.

\section{INTRODUCTION}

In a separate paper (Cellino et al. 2015a, hereinafter Paper I), we have performed an extensive analysis of the problem of finding satisfactory calibrations of several relations between different polarimetric parameters and the geometric albedo. Our analysis was based on using for calibration purposes a sample of asteroids for which we have reliable independent estimates of the albedo, based on accurate measurements of their size, reliable estimates of their absolute magnitude, and using the known relation linking the size, geometric albedo and absolute magnitude of an asteroid, namely:

$\log (D)=3.1236-0.2 H-0.5 \log \left(p_{V}\right)$,

where $D$ is the diameter expressed in $\mathrm{km}$ (assuming the object is spherical), $H$ is the absolute magnitude (in the Johnson $V$ band by

^E-mail: cellino@oato.inaf.it (AC); rgilhutton@ casleo.gov.ar (RG-H);
eft@psi.edu (EFT) definition) and $p_{V}$ is the geometric albedo (again, in the Johnson $V$ band). A list of asteroids suited for the purposes of calibration was published by Shevchenko \& Tedesco (2006), hereinafter S\&T (2006). In recent years we have performed campaigns of polarimetric observations of objects belonging to the S\&T (2006) list, in order to obtain for them accurate polarimetric measurements. Paper I presented the results of our analysis of these asteroids. By analysing the phase-polarization curves of the objects, namely the variation of the linear polarization $P_{r}$ as a function of the solar phase angle, ${ }^{1}$ we computed a variety of polarimetric parameters that are known to be diagnostic of albedo, including the polarimetric

\footnotetext{
${ }^{1} P_{r}$ is the degree of linear polarization with a sign that is defined to be positive when the plane of polarization is found to be perpendicular to the Sun - observer - target plane (scattering plane), and negative when the plane of polarization is parallel to the scattering plane. The solar phase angle, herein after referred to simply as the 'phase angle', is the angle between the directions to the Sun and to the observer, as seen from the target object.
} 
slope $h$ and the extreme value of negative polarization $P_{\min }$ (for an introduction to the basic notions of asteroid polarimetry, that we will not repeat here, see Paper I). In addition, we also considered some new polarimetric parameters, including the $p^{*}$ parameter suggested by Masiero et al. (2012), as well as a new parameter, named $\Psi$, that we introduced in Paper I.

In order to derive accurate values of the above polarimetric parameters from available data, in Paper I we focused our analysis on asteroids for which we have a good number of polarimetric measurements satisfactorily sampling the phase-polarization curves, and we made use of the following exponential-linear relation to fit the phase-polarization curves of the objects under scrutiny:

$P_{r}=A\left(\mathrm{e}^{-\alpha / B}-1\right)+C \alpha$,

where $\alpha$ is the phase angle expressed in degrees, and $A, B, C$ are parameters whose values have to be determined by means of bestfitting techniques. The above analytical representation has been found in the past to be well suited to fit phase-polarization curves (Muinonen et al. 2009). Note that this relation does not take into account the possible presence of a polarization surge at very small phase angles. This effect, found by Rosenbush et al. (2005) and Rosenbush et al. (2009) to be possibly present in the case of a couple of very high albedo asteroids, (64) Angelina and (44) Nysa, respectively, is rather negligible for the purposes of the present analysis. We do not analyse Angelina because we already did it in Paper I. We note also that the available measurements obtained for this object at very small phase angles have error bars too large to be accepted by our severe selection criteria adopted in Paper I. In the case of Nysa, which is analysed in this paper, the few measurements suggesting a surge of negative polarization at phase angles $<2^{\circ}$ do not seem to produce any important consequence on the overall fit of its phase-polarization curve, although we will see below that this asteroid seems to be rather peculiar in some aspects.

Using the exponential-linear relation, we were able to find suitable calibrations of several relations between the geometric albedo and polarimetric parameters, generally described in the form:

$\log \left(p_{V}\right)=A \log (w)+B$,

where $p_{V}$ is the geometric albedo in $V$ light and $w$ is one of several possible polarimetric parameters characterizing the morphology of available phase-polarization curves.

In Paper I, that was exclusively focused on the issue of the calibration of different albedo-polarization relations, we analysed 22 asteroids belonging to the S\&T (2006) list. However, we could also compute the most relevant polarimetric parameters for a larger number of other asteroids, not included in the above-mentioned list, because they were not suitable for the specific purposes of Paper I. In this paper, we present our results for the remaining objects. In particular, we consider all available polarimetric data, taken from different sources, including the PDS, ${ }^{2}$ and recent papers (Gil-Hutton \& Cañada-Assandri 2011, 2012; Cañada-Assandri, Gil-Hutton \& Benavidez 2012; Gil-Hutton, Cellino \& Bendjoya 2014). For each object, we computed different estimates of the albedos using the same methods used in Paper I.

Moreover, in this paper we also present a more extensive analysis of the distribution of different polarimetric parameters among the asteroid population, using all available data, and we also analyse

\footnotetext{
${ }^{2}$ Data available at http://pds.jpl.nasa.gov/ (files maintained by D.F. Lupishko and I.N. Belskaya).
}

some interesting relations between different polarimetric parameters. This was beyond the scope of the analysis performed in Paper I, but we investigate now such relations, including some that in the past were considered to be directly diagnostic of surface properties, including the typical sizes of regolith particles.

\section{POLARIZATION PARAMETERS AND GEOMETRIC ALBEDOS}

In Paper I (tables 2, 3, and 5) we listed a summary of several polarimetric parameters and corresponding albedo values obtained for 22 objects from S\&T (2006). These asteroids, for which we made an effort to obtain new polarimetric measurements, were chosen for the purposes of calibration of different possible albedo-polarization relations. Here, we consider an additional set of 64 asteroids that are not included in S\&T (2006). These asteroids were chosen from those that, in our judgment, have phase-polarization data of sufficiently good quality to derive the major polarimetric parameters with an accuracy high enough for use in the present study. In terms of requirements concerning data quality and coverage of the phasepolarization curves, we made our selection using the same criteria already adopted and described in Paper I.

Table 1 shows for these objects the polarimetric slope $h$, resulting from the computation of a simple linear fit of a minimum of five $P_{r}$ measurements obtained at phase angles larger than $14^{\circ}$, as well as some other parameters considered in Paper I. Apart from $h$, these parameters were obtained from fitting an exponential-linear fit (equation 2) to the whole phase-polarization curves. These parameters include another independent estimate of the polarimetric slope, that we called $h_{A B C}$, obtained as the first derivative of the exponential-linear curve computed at the inversion angle $\alpha_{\text {inv }}$; the extreme value of negative polarization $P_{\min }$; the $\boldsymbol{\Psi}$ parameter introduced in Paper I (defined as the difference between the values of $P_{r}$ formally corresponding to phase angles of $30^{\circ}$ and $10^{\circ}$, respectively, according to the best fit of equation 2 ), and the $p^{*}$ parameter defined by Masiero et al. (2012). The low associated uncertainty of the inversion angle $\alpha_{\text {inv }}$ comes also from the best fit of the exponential-linear relation described in Paper I.

The difference between the polarization slopes $h$ and $h_{A B C}$ consists only in the way they are computed. In Paper I, we showed that the use of $h$ or $h_{A B C}$ gives very similar solutions, apart from marginally better RMS deviations in the case of using $h$. So, in practical situations the use of either $h$ or $h_{A B C}$ is mainly dictated by the available polarimetric data.

It is important to note that in Paper I we considered two kinds of calibration of the slope-albedo and $P_{\min }$-albedo relations. The first was obtained by fitting all data available for all the objects of our data set. The second was obtained by removing from the analysis asteroids that according to S\&T (2006) have albedos lower than 0.08 . This was suggested by the evidence that the slope-albedo and (even more) the $P_{\min }$-albedo relation tend to saturate at low albedo. We showed that the linear best-fitting solutions obtained by excluding low-albedo asteroids from the analysis give smaller RMS deviations of the data, and should therefore be preferred, but only in narrower intervals of $h$ and $P_{\min }$, because at values of $h>0.25$ per cent $\mathrm{deg}^{-1}$ and $P_{\min }$ deeper than -1 per cent, there is an unsolvable ambiguity between objects of quite different albedo, but sharing the same values of $h$ (or $h_{A B C}$ ) and $P_{\min }$. We found also that the slope-albedo relation calibrated against asteroids of all albedos can still be used to derive a decent albedo estimate for any object, whereas the use of the $P_{\min }$-albedo relation calibrated against 
Table 1. Summary of the formal solutions for the polarimetric parameters for all asteroids not included in the S\&T (2006) list, for which we have a suitable coverage of the phase-polarization curves. Each asteroid is identified by its number. The second column gives the number $N_{\text {obs }}$ of polarimetric measurements used in the analysis. For the meaning of the other parameters, see the text. The same table for asteroids included in the S\&T (2006) list have been published in Paper I.

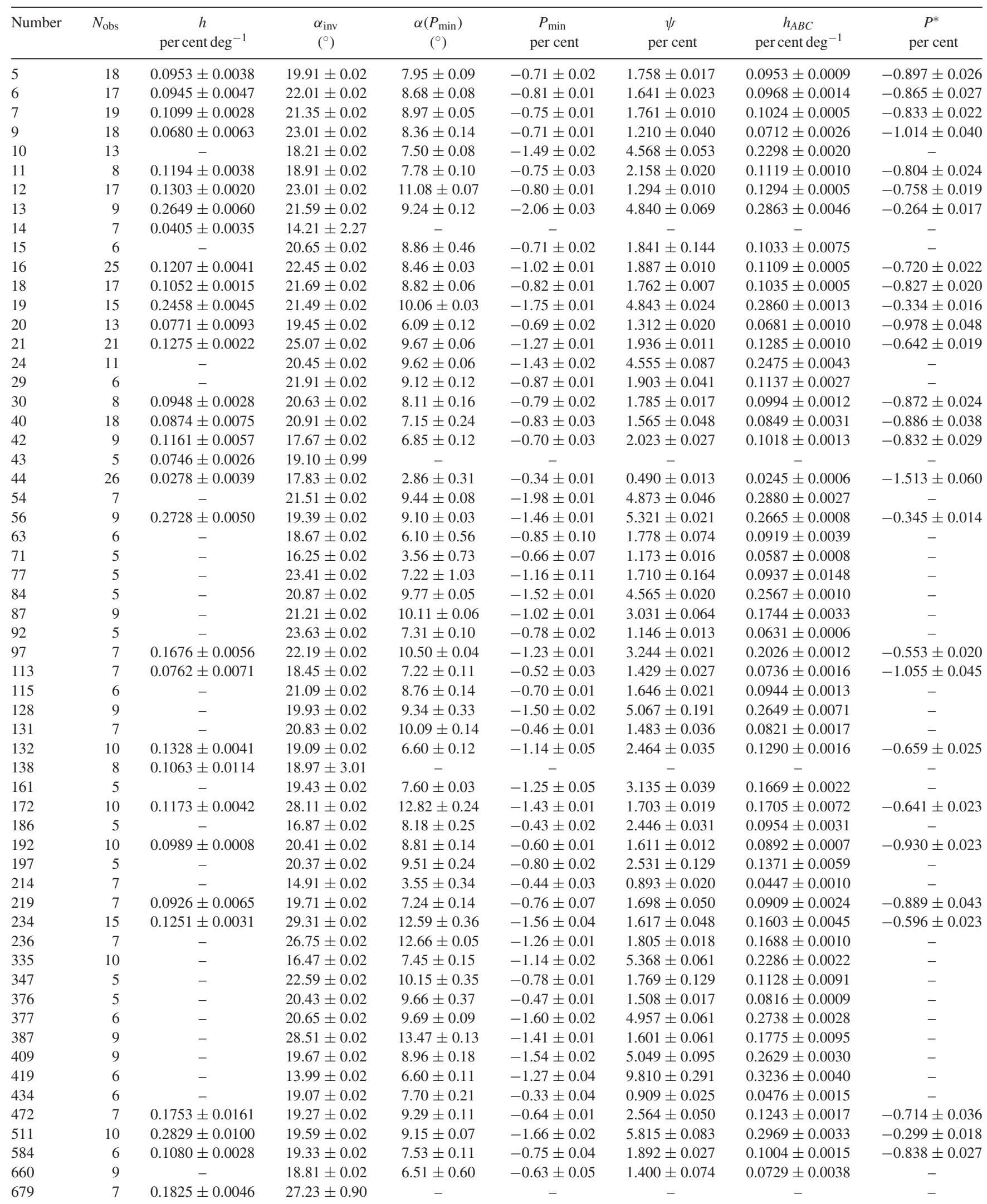


Table 1 continued

\begin{tabular}{|c|c|c|c|c|c|c|c|c|}
\hline Number & $N_{\text {obs }}$ & $\begin{array}{c}h \\
\text { per cent deg } \\
-1\end{array}$ & $\begin{array}{c}\alpha_{\text {inv }} \\
\left({ }^{\circ}\right)\end{array}$ & $\begin{array}{c}\alpha\left(P_{\min }\right) \\
\left({ }^{\circ}\right)\end{array}$ & $\begin{array}{c}P_{\min } \\
\text { per cent }\end{array}$ & $\begin{array}{c}\psi \\
\text { per cent }\end{array}$ & $\begin{array}{c}h_{A B C} \\
\text { per cent deg } \\
-1\end{array}$ & $\begin{array}{c}P^{*} \\
\text { per cent }\end{array}$ \\
\hline 796 & 7 & $0.1145 \pm 0.0032$ & $21.07 \pm 0.02$ & $6.71 \pm 0.33$ & $-1.21 \pm 0.10$ & $2.122 \pm 0.062$ & $0.1132 \pm 0.0017$ & $-0.692 \pm 0.034$ \\
\hline 980 & 6 & - & $29.11 \pm 0.02$ & $12.20 \pm 0.14$ & $-1.24 \pm 0.01$ & $1.317 \pm 0.049$ & $0.1231 \pm 0.0068$ & - \\
\hline 1021 & 10 & - & $15.63 \pm 0.02$ & $7.51 \pm 0.10$ & $-0.71 \pm 0.02$ & $4.673 \pm 0.042$ & $0.1675 \pm 0.0017$ & - \\
\hline 2867 & 6 & - & $17.15 \pm 0.02$ & $4.11 \pm 0.62$ & $-0.41 \pm 0.04$ & $0.737 \pm 0.020$ & $0.0370 \pm 0.0010$ & - \\
\hline
\end{tabular}

asteroids of all albedos should not be used, since the resulting errors on the derived albedos are exceedingly high.

In Table 2, we list the albedo values obtained from the values of different polarimetric parameters considered in Paper I. We give, whenever possible, two values of albedo obtained from the $h$ slope, one corresponding to the calibration of the slope-albedo relation using all calibration asteroids considered in Paper I, and one corresponding to the calibration obtained considering only the objects having albedo larger than 0.08 . The latter albedo value is given only for asteroids having $h<0.25$ per cent $\mathrm{deg}^{-1}$. As for the albedo computed using $P_{\min }$, we use only the calibration obtained in Paper I for asteroids having albedo larger than 0.08 , and we list the corresponding albedo only for asteroids having $P_{\min }$ not reaching 1 per cent. In so doing, we are following our own recommendations as explained in Paper I. In the case of $h_{A B C}$ and $\Psi$, the resulting albedo values given in Table 2 are based on the whole set of calibration asteroids, including also low-albedo objects. In Paper I we showed that in the case of $\Psi$-based albedos, the resulting values are generally very reliable. In the case of $h_{A B C}$, however, it should be better to exclude from the calibration the asteroids having albedo smaller than 0.08 (as in the case of $h$ just mentioned). The albedo values listed in Table 2 corresponding to $h_{A B C}$ must therefore be taken with some caveat, since some overestimation of albedos, specially for high-albedo objects, is likely present.

From Table 2, we can see that most albedo values are obtained from computation of the $h_{A B C}$ and $\Psi$ polarimetric parameters, obtained from a fit of the phase-polarization curve using equation (2). In general, the agreement among the albedos obtained using different polarimetric parameters is quite good. This confirms that, depending on the available data, reliable albedo values can be obtained even from a fairly small number of polarimetric measurements. The best polarimetric parameter to be used depends case by case upon the available data, and we have outlined in Paper I how to proceed in practical situations.

The albedo values obtained from the $\Psi$ parameter tend to vary in a more limited interval with respect to what we find using other polarimetric parameters. This is particularly true for the highest albedo objects, (44) Nysa, (214) Aschera, and (2867) Steins, included in our sample. These asteroids belong to the old $E$ class defined by Tholen (Tholen \& Barucci 1989); in the more recent classification by Bus \& Binzel (2002) they are classified as Xc. There is some problem concerning in particular (44) Nysa. As shown in Table 2, we find extremely high values, up to 0.9 or even above, using the slope-albedo relation calibrated against all asteroids considered in Paper I, regardless of their albedo. By using calibrations obtained by dropping low-albedo asteroids, the resulting albedo tends to decrease down to 0.6. This was one of the main reasons in Paper I to compute alternative calibrations based on the exclusion of the darkest calibration objects. In the case of the $p^{*}$-albedo relation, we obtain for Nysa a still high value around 0.8 . A slightly more moderate value, around 0.7 , is found using the $\Psi$ parameter. The

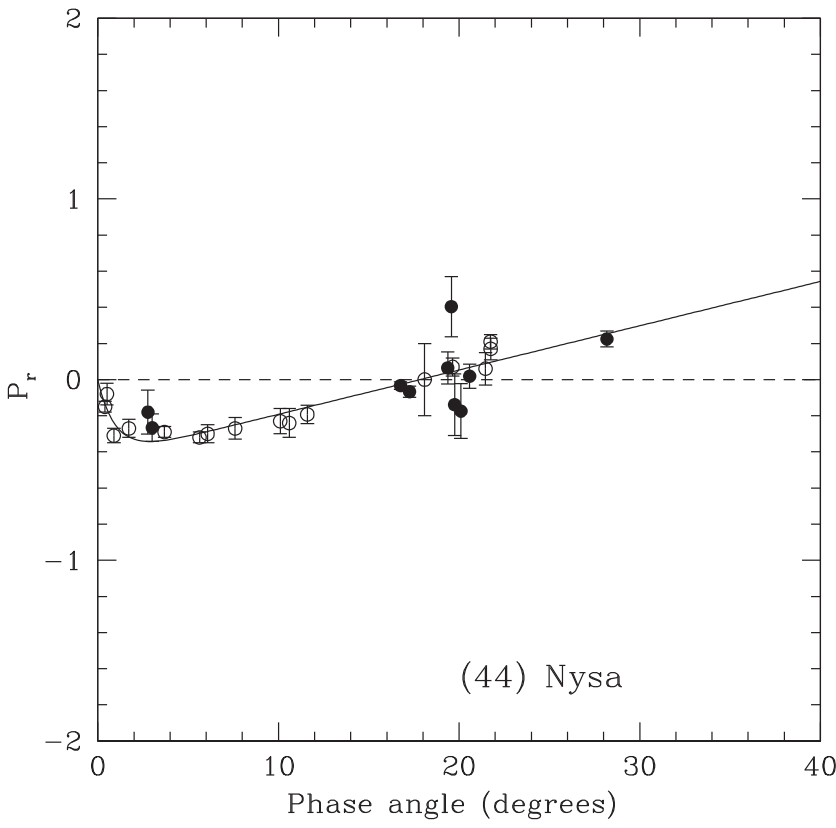

Figure 1. The phase-polarization curve of the high-albedo asteroid (44) Nysa. In this and other figures, full symbols indicate measurements taken at the Complejo Astronomico El Leoncito (CASLEO, Argentina) observatory and recently published by Gil-Hutton et al. (2014). Open symbols are other measurements taken from the literature. The solid curve is the best-fitting curve using the exponential-linear representation (Equation 2).

extremely shallow polarimetric slope of Nysa is shown in Fig. 1. In this figure, note also the very low value of the phase angle corresponding to $P_{\min }$. The albedos of (214) Aschera and (2867) Steins, turn out to be much lower than in the case of (44) Nysa. Also for another $E$-class asteroid, (434) Hungaria, that is now classified as Xe by Bus \& Binzel (2002), we find a much more moderate albedo value, slightly above 0.4 . In general terms, $E$-class asteroids are those for which the choice of the polarimetric parameter chosen to derive the albedo makes the most difference, and for which it is highly recommended to use calibrations of the polarimetric slope and of $P_{\min }$ that are computed by excluding low-albedo objects from the computation. In other words, albedo values in columns 2 and 4 of Table 2 should not be used for $E$-class objects. In this way, with the notable exception of (44) Nysa, the albedo values obtained are generally in reasonable mutual agreement, ranging approximately between 0.4 and 0.5 , a range that one might expect corresponds to a real variation among the objects of this class.

It is also interesting to note in Table 2 the high albedo values found for the $A$-class asteroid (863) Benkoela, ranging from 0.4 to 0.7 , depending on the adopted polarimetric parameter. This is the only example of $A$-class objects in our sample. Further observations of other members of this fairly rare class, which is thought to have 
Table 2. Geometric albedo values $p_{V}$ for all asteroids not belonging to the S\&T (2006) list, for which we have polarimetric observations suited to derive the albedo using one or more of the relations explained in Paper I. The columns marked as 'no low- $p_{V}$ ' refer to calibrations of the $h-p_{V}$ and $P_{\min }-p_{V}$ relations computed using asteroids having $p_{V}>0.08$, only (see text).

\begin{tabular}{|c|c|c|c|c|c|c|}
\hline Number & $p_{V}(h)$ & $\begin{array}{c}p_{V}(h) \\
\text { (no low- } p_{V} \text { ) }\end{array}$ & $p_{V}\left(h_{A B C}\right)$ & $\begin{array}{c}p_{V}\left(P_{\min }\right) \\
\left(\text { no low- } p_{V}\right)\end{array}$ & $p_{V}(\Psi)$ & $p_{V}\left(p^{*}\right)$ \\
\hline 5 & $0.226 \pm 0.023$ & $0.224 \pm 0.030$ & $0.206 \pm 0.016$ & $0.219 \pm 0.007$ & $0.200 \pm 0.007$ & $0.222 \pm 0.020$ \\
\hline 7 & $0.193 \pm 0.018$ & $0.200 \pm 0.025$ & $0.189 \pm 0.015$ & $0.208 \pm 0.025$ & $0.199 \pm 0.007$ & $0.195 \pm 0.016$ \\
\hline 9 & $0.328 \pm 0.047$ & $0.293 \pm 0.046$ & $0.286 \pm 0.027$ & $0.220 \pm 0.006$ & $0.289 \pm 0.013$ & $0.283 \pm 0.032$ \\
\hline 10 & - & - & $0.075 \pm 0.005$ & - & $0.078 \pm 0.004$ & - \\
\hline 11 & $0.176 \pm 0.017$ & $0.187 \pm 0.023$ & $0.171 \pm 0.013$ & $0.208 \pm 0.008$ & $0.163 \pm 0.006$ & $0.184 \pm 0.015$ \\
\hline 14 & $0.584 \pm 0.087$ & $0.444 \pm 0.076$ & - & - & - & - \\
\hline 15 & - & - & $0.187 \pm 0.021$ & $0.219 \pm 0.008$ & $0.191 \pm 0.016$ & - \\
\hline 16 & $0.173 \pm 0.016$ & $0.185 \pm 0.023$ & $0.173 \pm 0.013$ & - & $0.186 \pm 0.006$ & $0.154 \pm 0.012$ \\
\hline 18 & $0.202 \pm 0.019$ & $0.207 \pm 0.026$ & $0.187 \pm 0.014$ & $0.194 \pm 0.004$ & $0.199 \pm 0.007$ & $0.192 \pm 0.016$ \\
\hline 19 & $0.079 \pm 0.006$ & $0.105 \pm 0.011$ & $0.059 \pm 0.003$ & - & $0.073 \pm 0.003$ & $0.069 \pm 0.004$ \\
\hline 20 & $0.285 \pm 0.047$ & $0.265 \pm 0.044$ & $0.301 \pm 0.026$ & $0.225 \pm 0.008$ & $0.266 \pm 0.009$ & $0.263 \pm 0.033$ \\
\hline 40 & $0.248 \pm 0.033$ & $0.240 \pm 0.036$ & $0.234 \pm 0.021$ & $0.191 \pm 0.007$ & $0.224 \pm 0.010$ & $0.217 \pm 0.023$ \\
\hline 42 & $0.181 \pm 0.019$ & $0.191 \pm 0.025$ & $0.191 \pm 0.015$ & $0.221 \pm 0.009$ & $0.174 \pm 0.006$ & $0.194 \pm 0.017$ \\
\hline 43 & $0.296 \pm 0.031$ & $0.272 \pm 0.038$ & - & - & - & - \\
\hline 44 & $0.886 \pm 0.177$ & $0.599 \pm 0.122$ & $0.965 \pm 0.108$ & $0.412 \pm 0.024$ & $0.704 \pm 0.030$ & $0.791 \pm 0.013$ \\
\hline 54 & - & - & $0.058 \pm 0.003$ & - & $0.073 \pm 0.003$ & - \\
\hline 56 & $0.070 \pm 0.005$ & - & $0.064 \pm 0.004$ & - & $0.067 \pm 0.003$ & $0.071 \pm 0.004$ \\
\hline 63 & - & - & $0.214 \pm 0.020$ & $0.188 \pm 0.019$ & $0.197 \pm 0.010$ & - \\
\hline 71 & - & - & $0.357 \pm 0.032$ & $0.234 \pm 0.022$ & $0.298 \pm 0.010$ & - \\
\hline 77 & - & - & $0.210 \pm 0.041$ & - & $0.205 \pm 0.021$ & - \\
\hline 84 & - & - & $0.066 \pm 0.004$ & - & $0.078 \pm 0.004$ & - \\
\hline 87 & - & - & $0.103 \pm 0.007$ & - & $0.117 \pm 0.005$ & - \\
\hline 92 & - & - & $0.329 \pm 0.029$ & $0.201 \pm 0.006$ & $0.304 \pm 0.010$ & - \\
\hline 172 & $0.179 \pm 0.017$ & $0.189 \pm 0.024$ & $0.106 \pm 0.009$ & - & $0.206 \pm 0.007$ & $0.131 \pm 0.010$ \\
\hline 186 & - & - & $0.205 \pm 0.018$ & $0.341 \pm 0.022$ & $0.144 \pm 0.005$ & - \\
\hline 192 & $0.216 \pm 0.020$ & $0.217 \pm 0.028$ & $0.222 \pm 0.018$ & $0.254 \pm 0.009$ & $0.218 \pm 0.007$ & $0.238 \pm 0.021$ \\
\hline 197 & - & - & $0.136 \pm 0.012$ & $0.198 \pm 0.006$ & $0.139 \pm 0.009$ & - \\
\hline 214 & - & - & $0.487 \pm 0.047$ & $0.334 \pm 0.022$ & $0.390 \pm 0.015$ & - \\
\hline 219 & $0.233 \pm 0.028$ & $0.229 \pm 0.032$ & $0.217 \pm 0.018$ & $0.207 \pm 0.016$ & $0.207 \pm 0.009$ & $0.219 \pm 0.024$ \\
\hline 234 & $0.167 \pm 0.015$ & $0.180 \pm 0.022$ & $0.114 \pm 0.009$ & - & $0.217 \pm 0.009$ & $0.119 \pm 0.009$ \\
\hline 236 & - & - & $0.107 \pm 0.007$ & - & $0.194 \pm 0.007$ & - \\
\hline 335 & - & - & $0.076 \pm 0.005$ & - & $0.066 \pm 0.003$ & - \\
\hline 347 & - & - & $0.170 \pm 0.020$ & $0.201 \pm 0.005$ & $0.198 \pm 0.016$ & - \\
\hline 376 & - & - & $0.245 \pm 0.020$ & $0.316 \pm 0.013$ & $0.232 \pm 0.008$ & - \\
\hline 377 & - & - & $0.062 \pm 0.004$ & - & $0.072 \pm 0.003$ & - \\
\hline 387 & - & - & $0.101 \pm 0.009$ & - & $0.219 \pm 0.011$ & - \\
\hline 409 & - & - & $0.065 \pm 0.004$ & - & $0.070 \pm 0.004$ & - \\
\hline 419 & - & - & $0.051 \pm 0.003$ & - & $0.037 \pm 0.002$ & - \\
\hline 434 & - & - & $0.453 \pm 0.045$ & $0.423 \pm 0.044$ & $0.383 \pm 0.015$ & - \\
\hline 472 & $0.115 \pm 0.015$ & $0.137 \pm 0.018$ & $0.152 \pm 0.011$ & $0.238 \pm 0.007$ & $0.138 \pm 0.006$ & $0.152 \pm 0.015$ \\
\hline 511 & $0.067 \pm 0.005$ & - & $0.056 \pm 0.003$ & - & $0.061 \pm 0.003$ & $0.065 \pm 0.004$ \\
\hline 584 & $0.196 \pm 0.019$ & $0.202 \pm 0.026$ & $0.194 \pm 0.015$ & $0.208 \pm 0.010$ & $0.186 \pm 0.007$ & $0.197 \pm 0.017$ \\
\hline 660 & - & - & $0.279 \pm 0.029$ & $0.242 \pm 0.017$ & $0.250 \pm 0.015$ & - \\
\hline 679 & $0.110 \pm 0.009$ & $0.133 \pm 0.015$ & - & - & - & - \\
\hline 796 & $0.184 \pm 0.017$ & $0.193 \pm 0.024$ & $0.169 \pm 0.013$ & - & $0.166 \pm 0.007$ & $0.146 \pm 0.013$ \\
\hline
\end{tabular}


Table 2. continued

\begin{tabular}{|c|c|c|c|c|c|c|}
\hline Number & $p_{V}(h)$ & $\begin{array}{c}p_{V}(h) \\
\text { (no low- } p_{V} \text { ) }\end{array}$ & $p_{V}\left(h_{A B C}\right)$ & $\begin{array}{c}p_{V}\left(P_{\min }\right) \\
\left(\text { no low- } p_{V}\right)\end{array}$ & $p_{V}(\Psi)$ & $p_{V}\left(p^{*}\right)$ \\
\hline 863 & - & - & $0.545 \pm 0.057$ & $0.682 \pm 0.053$ & $0.372 \pm 0.019$ & - \\
\hline 1021 & - & - & $0.108 \pm 0.007$ & $0.219 \pm 0.008$ & $0.076 \pm 0.004$ & - \\
\hline 2867 & - & - & $0.604 \pm 0.062$ & $0.350 \pm 0.033$ & $0.471 \pm 0.019$ & - \\
\hline
\end{tabular}

a composition dominated by olivine, are needed to confirm this preliminary result.

When looking at the data displayed in Table 2, one should take into account that this table lists also a number of so-called Barbarian objects, which are known to exhibit peculiar polarimetric properties, and in particular a very wide width of the negative polarization branch, up to about $30^{\circ}$ in phase (Cellino et al. 2006; Gil-Hutton et al. 2008; Masiero \& Cellino 2009). For these objects, it is likely that the peculiar morphology of the phase-polarization curve can prevent us from deriving the albedo using the same polarimetric parameters developed for normal asteroids. Asteroids (234) Barbara (the prototype of the Barbarian class), (172) Baucis, (236) Honoria, (387) Aquitania, and (980) Anacostia, included in Table 2, are all Barbarians. For them, we see significant differences in the albedo values derived using different polarimetric parameters. The albedos derived using the $\Psi$ parameter, tend to have values around 0.20 , whereas in the case of using $h_{A B C}$ the corresponding value tends to be around 0.10. In both cases, (980) Anacostia seems to have a higher albedo than the other Barbarians in our sample.

If we exclude Barbarians, we see that the albedo values derived using different parameters show a remarkable consistency. We conclude therefore that it may be risky to try and derive albedos for Barbarian objects using the conventional polarimetric parameters, due to their unusual polarimetric behaviour. The situation is much more promising for the vast majority of 'normal' asteroids.

\section{THE DISTRIBUTION OF POLARIMETRIC PARAMETERS}

The availability of a data set of phase-polarization curves of sufficiently good quality to obtain best-fitting representations using the exponential-linear relation also allows us to make some updated investigations of the polarimetric behaviour of different classes of asteroids, not limited to the determination of the geometric albedo.

In particular, we can analyse the distributions of the inversion angle $\alpha_{\mathrm{inv}}$, and of the phase angle corresponding to $P_{\min }$, as shown in Figs 2 and 4, respectively.

Since the early days of asteroid polarimetry it has been known that the inversion angle occurs at phase angles close to $20^{\circ}$. In recent years, however, some important exceptions have been found, as shown in Fig. 2. By looking at the low-end distribution of $\alpha_{\text {inv }}$, there are two objects characterized by an inversion angle around $14^{\circ}$. In the case of asteroid (14) Irene, we cannot draw any conclusion because its phase-polarization curve is not of a very good quality and new observations are needed to better understand its true behaviour. The case of (419) Aurelia, conversely, is much more interesting. This asteroid belongs to the old $F$ taxonomic class identified by Gradie \& Tedesco (1982; see also Tedesco et al. 1989). (419) Aurelia is no longer identified as an $F$ class in more recent taxonomic classifications based on reflectance spectra that no longer cover the blue part of the spectrum. Asteroids previously classified as $F$ are now included in the modern $B$ class (Bus \& Binzel 2002).

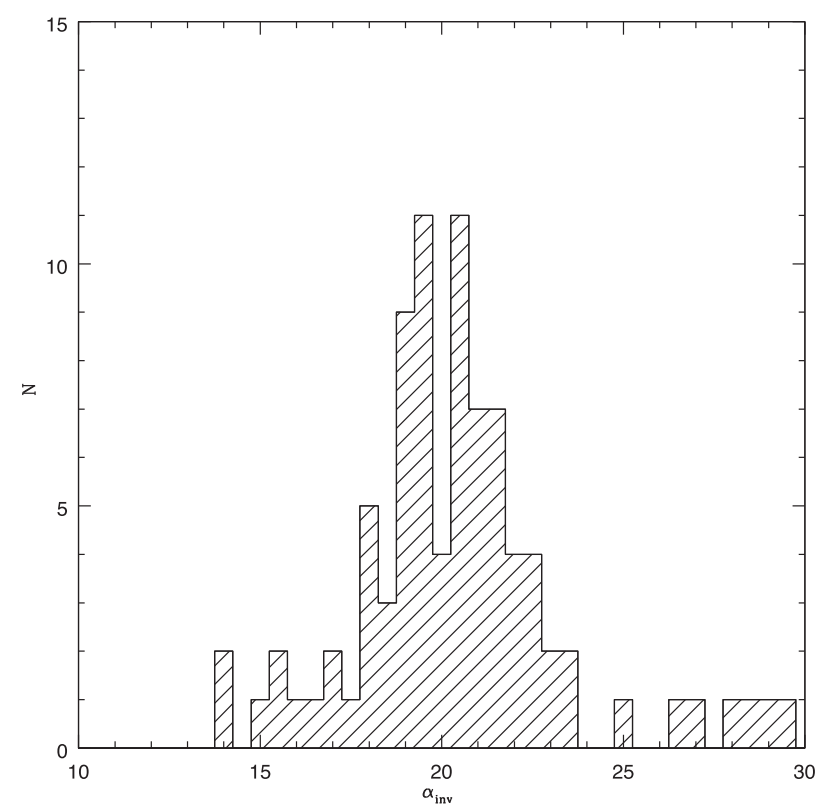

Figure 2. Histogram of the inversion angle $\alpha_{\text {inv }}$ for all asteroids considered in this paper and in Paper I.

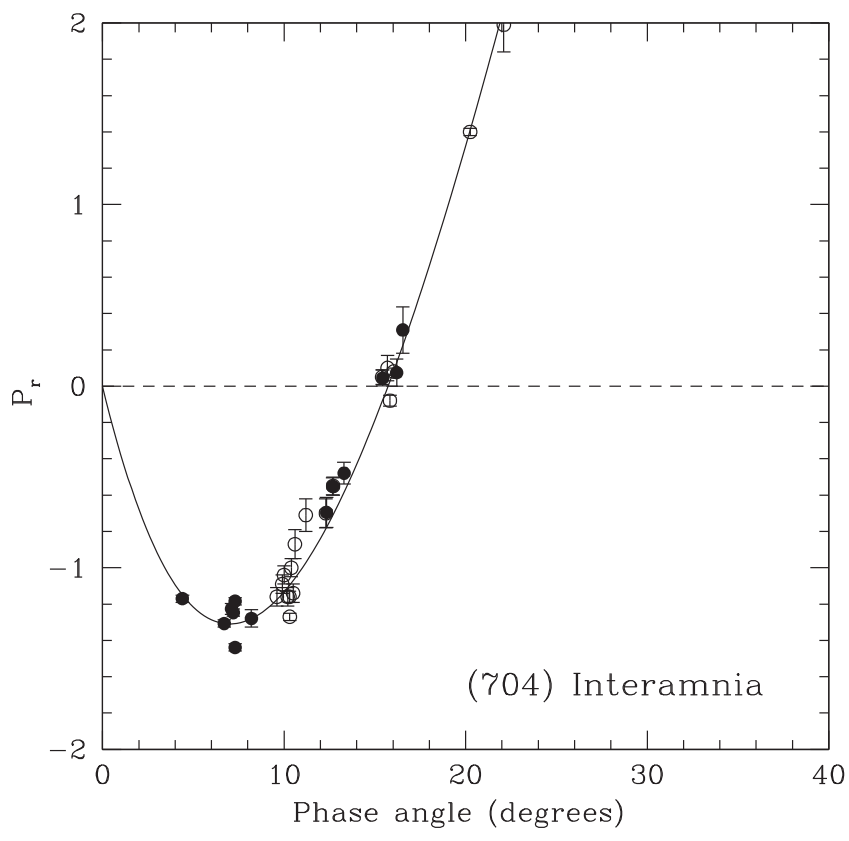

Figure 3. The phase-polarization curve of the low-albedo asteroid (704) Interamnia. Full symbols indicate measurements taken at the CASLEO observatory. Open symbols are other measurements taken from the literature. The solid curve is the best-fitting curve using the exponential-linear representation (equation 2). 


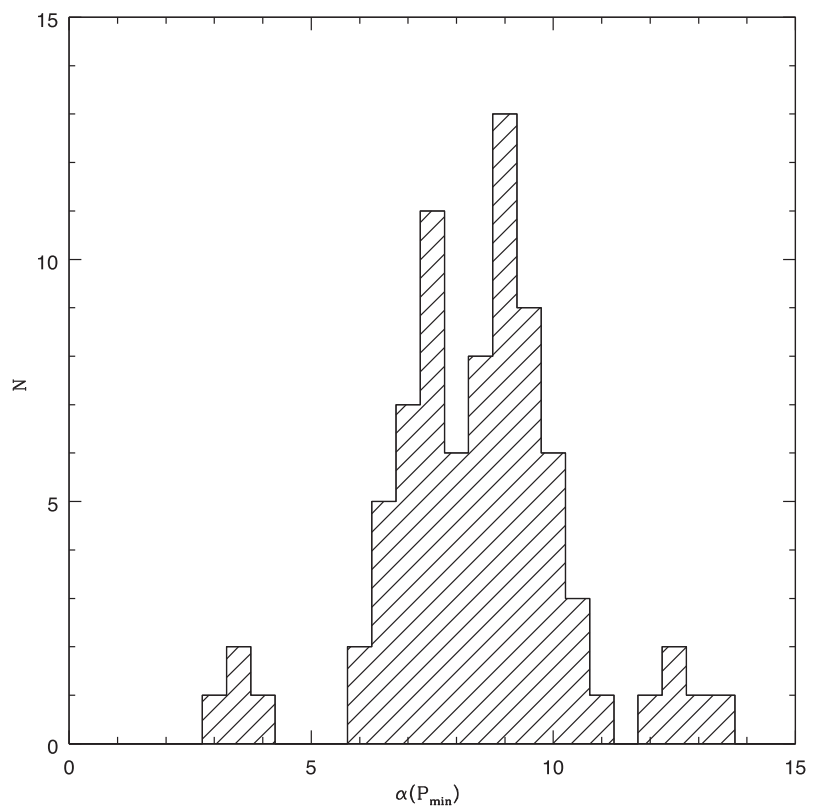

Figure 4. Histogram of the values of phase angle corresponding to $P_{\min }$ for all asteroids considered in this paper and in Paper I.

We know, however, that the original $F$-class asteroids can be distinguished based on their polarimetric properties. In particular, these asteroids are characterized by small values of $\alpha_{\text {inv }}$ (Belskaya et al. 2005). In addition to Aurelia, three other $F$-class asteroids in our sample show low values of $\alpha_{\text {inv }}$, around $16^{\circ}$. They are (335) Roberta, (704) Interamnia (analysed in Paper I), and (1021) Flammario, although in the case of Flammario the available polarimetric data are noisy. The excellent phase-polarization curve of (704) Interamnia, one of the calibration asteroids used in Paper I, is shown here in Fig. 3.

In addition to the above-mentioned $F$-class asteroids, another asteroid, (214) Aschera, exhibits an inversion angle of about $15^{\circ}$. This is a very high albedo asteroid, and its properties will be discussed below. A few other objects exhibit relatively low values of $\alpha_{\text {inv }}$ around $18^{\circ}$. We have seen, however, that for them we still need additional observations to better characterize their phase-polarization curves.

At the other end of the distribution of $\alpha_{\text {inv }}$, we see some objects characterized by values well above $20^{\circ}$. These are the Barbarian asteroids already mentioned above. Four of them, (172) Baucis, (234) Barbara, (387) Aquitania, and (980) Anacostia have inversion angles above $28^{\circ}$. (236) Honoria and (679) Pax have slightly lower inversion angles around $27^{\circ}$. The single object exhibiting an inversion angle of about $25^{\circ}$ is (21) Lutetia. This object is one of the two asteroids observed by the Rosetta probe (the other being (2867) Steins, also included in our tables). Lutetia has fairly unusual properties. It was classified in the past as an $M$-class, possibly metal-rich, asteroid (Tholen \& Barucci 1989), but the observations performed before and during the Rosetta fly-by, using different techniques, have shown that the composition of this asteroid seems to be more compatible with that of some classes of primitive meteorites (Coradini et al. 2011). The high value of the inversion angle of (21) Lutetia confirms that the surface of this asteroid is unusual.

The distribution of the inversion angle of polarization among asteroids appears to be today much wider and interesting than in the past. The $\alpha_{\text {inv }}$ parameter appears to be useful to distinguish classes of asteroids with unusual surface properties. In the case of
Barbarians, there are reasons to believe that these objects might be the remnants of the first generation of planetesimals accreted in the first few $10^{5} \mathrm{yr}$ of our Solar system's history (see Cellino et al. 2014 , and references therein). In the case of objects exhibiting small values of $\alpha_{\text {inv }}$, it is interesting to note that this property, typical of asteroids belonging to the old $F$ class of Gradie \& Tedesco (1982), has been found to be shared also by a few cometary nuclei (Bagnulo et al. 2011). This interesting result tends to strengthen other pieces of evidence of a possible link between $F$-class asteroids and comets, already suggested by the fact that comet Wilson Hurrington was first discovered as an asteroid (numbered 4015), classified as $C F$ and another $F$-class asteroid, (3200) Phaeton, is known to be the source of the Geminid meteors (Chamberlin et al. 1996, and references therein).

The distribution of the phase angle corresponding to $P_{\min }$, $\alpha\left(P_{\min }\right)$, is shown in Fig. 4 . We see a confirmation of the fact that $P_{\min }$ is mostly found at phase angles between $7^{\circ}$ and $10^{\circ}$. A few exceptions exist, however. We find four asteroids having $\alpha\left(P_{\min }\right)$ between $3^{\circ}$ and $4^{\circ}$, namely (44) Nysa, (71) Niobe, (214) Aschera, and (2867) Steins. We have already seen that (44), (214), and (2867) are high-albedo asteroids belonging to the old $E$ class. As for (71) Niobe, it is classified as $\mathrm{Xe}$ by Bus \& Binzel (2002). Its albedo, according to our results listed in Table 2, is high, though not as extreme as those of Nysa, Aschera, and Steins. Its phase-polarization curve, however, does not include data at phase angles less than about $7^{\circ}$, so the formal value of $\alpha\left(P_{\min }\right)$ listed in Table 1 for this asteroid is still very uncertain, and could be considerably wrong. It seems, anyway, that a very low value of $\alpha\left(P_{\min }\right)$ can be a common feature among high-albedo asteroids, although some of them, like the $\mathrm{Xe}$ class (434) Phocaea and the $A$-class (863) Benkoela do not share this property. It is possible that for the highest albedo asteroids, having very shallow polarimetric slopes, determination of the inversion angle and the phase angle of $P_{\min }$ might turn out to be more uncertain than the results presented here. We have already seen in Fig. 1, however, that the low value of $\alpha\left(P_{\min }\right)$ for (44) Nysa, seems well defined, and does not appear to be affected by a possible polarization opposition effect. Though not so densely sampled, the same can be seen for the phase-polarization curve of (214) Aschera, shown in Fig. 5. We note that very low values of $\alpha\left(P_{\min }\right)$ are known also for two Centaur objects, (2060) Chiron and (10199) Chariklo, and for the TNO object (5145) Pholus (Bagnulo et al. 2006; Belskaya et al. 2010). A fundamental difference with respect to high-albedo asteroids is that for objects at high heliocentric distances $P_{\min }$ is much deeper, and suggests low-albedo surfaces.

At the other end of the $\alpha\left(P_{\min }\right)$ distribution, there are five asteroids having phase angle of $P_{\min }$ between $12^{\circ}$ and $14^{\circ}$. These are the five Barbarian asteroids discussed above. Having a very wide negative polarization branch, it is not too surprising that these objects tend also to have $P_{\min }$ at larger phase angles than usual, and this feature might be related to the same surface properties that determine the wide negative polarization branch. We note, however, that (21) Lutetia, which also exhibits a large value of the inversion angle, is a perfectly normal asteroid as far as $\alpha\left(P_{\min }\right)$ is concerned.

\section{RELATIONS BETWEEN POLARIMETRIC PARAMETERS}

In Fig. 6, we present an $\alpha\left(P_{\min }\right)-h_{A B C}$ plot for all the asteroids considered in this paper (Table 1) and including also those considered in Paper I. The meaning of this figure is clear if we consider that the polarimetric slope $\left(h\right.$, or, as in this case, $h_{A B C}$, to consider a 


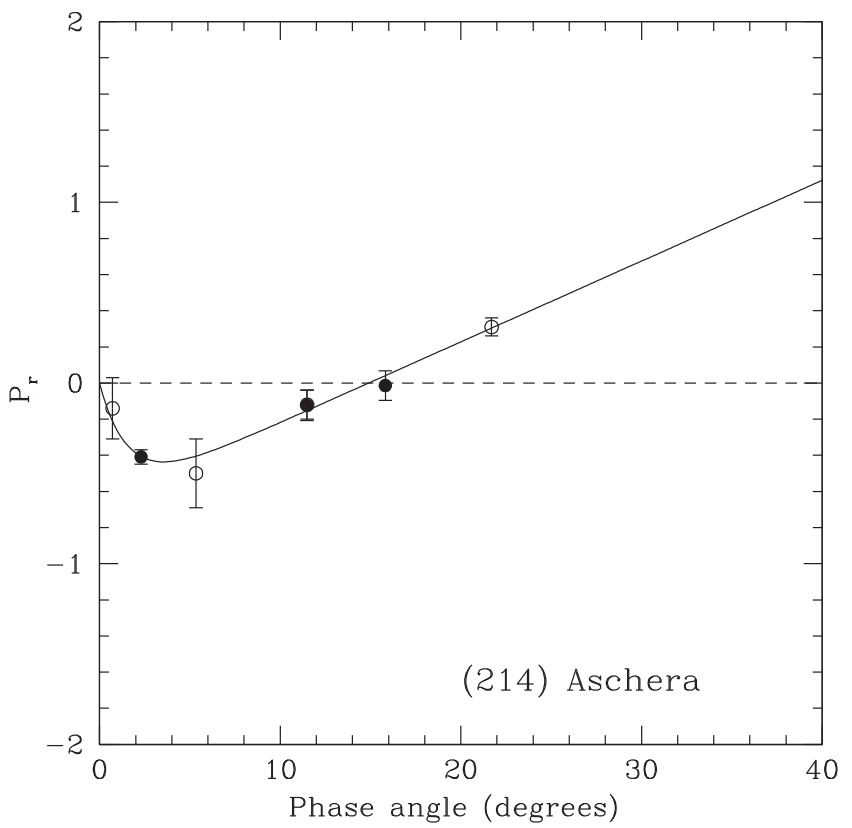

Figure 5. The phase-polarization curve of the high-albedo asteroid (214) Aschera. Full symbols indicate measurements taken at the CASLEO observatory. Open symbols are other measurements taken from the literature. Two different measurements obtained by different authors at a phase angle of 11.5 perfectly overlap in this plot. The solid curve is the best-fitting curve using the exponential-linear representation (equation 2).

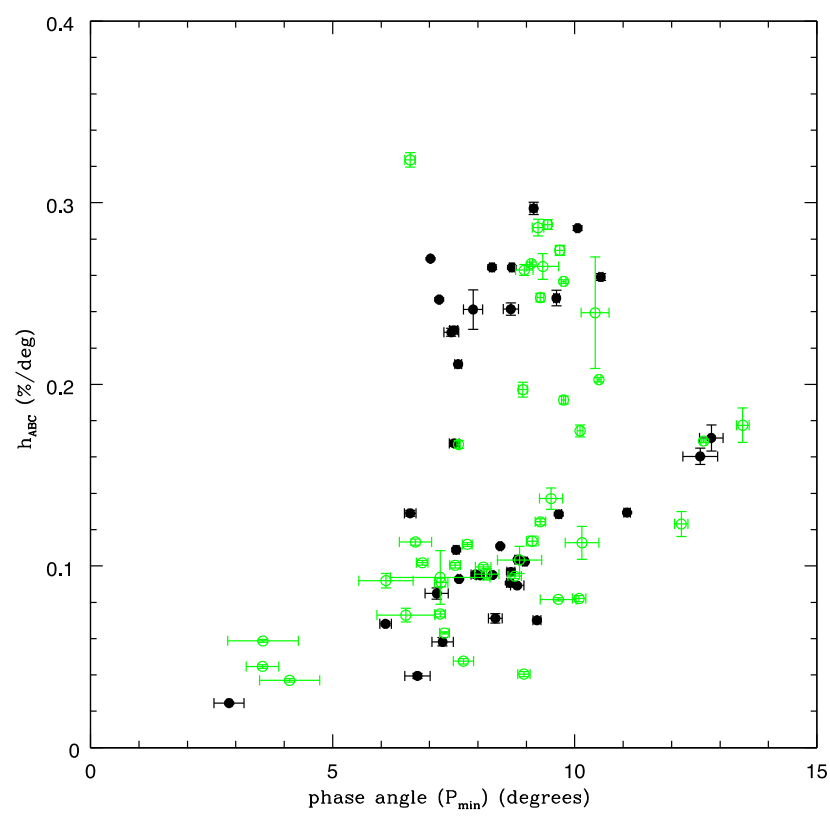

Figure 6. The $h_{A B C}$ polarimetric slope versus phase angle of $P_{\min }$ for the whole sample of asteroids considered in Tables 1 and 2 and Paper I. Note that higher values of $h_{A B C}$ correspond to lower values of albedo. Objects having fewer than 10 polarimetric measurements are indicated by open, green symbols.

larger number of objects) is diagnostic of the albedo. In particular, low-albedo asteroids have higher values of $h_{A B C}$, and vice versa.

From the figure, we see that the objects tend to split into two groups, characterized by different average values of slope (i.e. albedo). This is expected considering that the main belt population

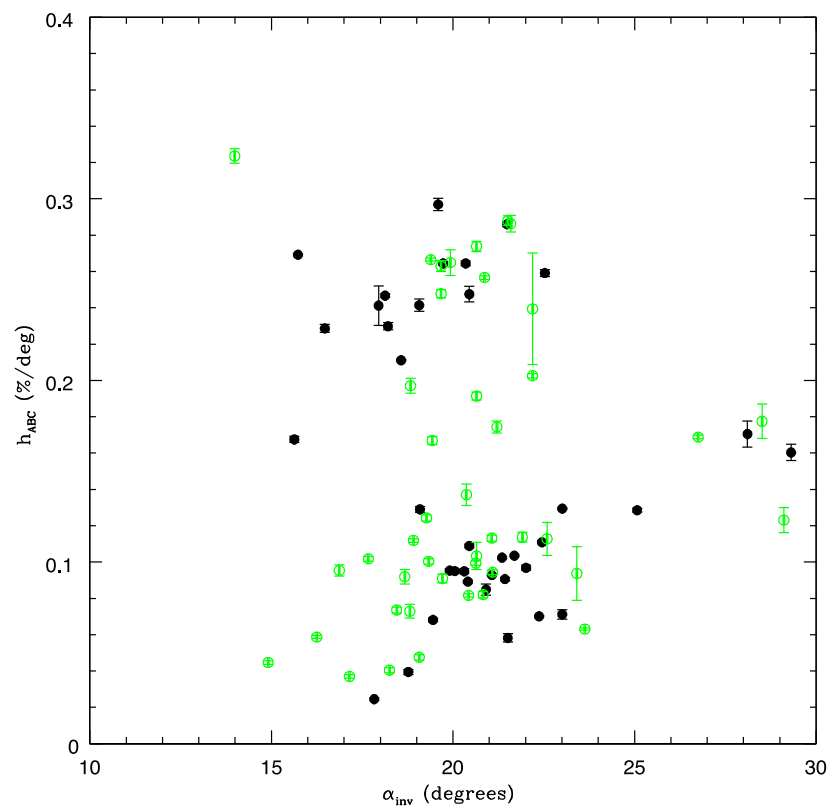

Figure 7. The $h_{A B C}$ polarimetric slope versus inversion angle $\alpha_{\text {inv }}$ for the whole sample of asteroids considered in Table 1 and Paper I. Asteroids for which we have fewer than 10 polarimetric measurements are indicated by open, green symbols.

is dominated by two superclasses, namely the moderate-albedo $S$ class, and the low-albedo $C$ class.

In the lower part of the plot shown in Fig. 6, corresponding to moderate to high-albedo objects, we see a general trend of decreasing albedo for increasing $\alpha\left(P_{\min }\right)$. A similar trend, but slightly less pronounced, may be seen also in the upper part of the plot. Lowalbedo objects (having higher values of the polarimetric slope $h_{A B C}$ ), tend to display values of $\alpha\left(P_{\min }\right)$ which look more confined. Asteroids having $\alpha\left(P_{\min }\right)$ below $6^{\circ}$ are found only among high-albedo objects, as already seen in Section 3. Most asteroids in Fig. 6 are located in the region between about $7^{\circ}$ and $10^{\circ}$ of $\alpha\left(P_{\min }\right)$. The righthand part of the plot is occupied by Barbarian asteroids, which tend to cluster at values of $h_{A B C}$ intermediate between those of low- and high-albedo objects.

In Fig. 7, we show a plot of the $h_{A B C}$ polarimetric slope versus the inversion angle $\alpha_{\text {inv }}$. Again, we can see a clear splitting between low-albedo asteroids and the rest of the population. In both groups, there is a trend for an increase of $h_{A B C}$ (equivalent to a decrease of the albedo) for increasing $\alpha_{\text {inv }}$. This behaviour is possibly less sharp among low-albedo objects, and $F$-class asteroids clearly do not follow this trend, with (419) Aurelia occupying the most extreme top-left location in the plot. All asteroids, independent of their albedo, tend to share the same interval of inversion angles, except for the Barbarians, which occupy the high-end of the $\alpha_{\text {inv }}$ range. Interesting enough, the location of the Barbarians in this plot tends to correspond to an extrapolation towards larger values of $\alpha_{\text {inv }}$ of the relation between $\alpha_{\text {inv }}$ and $h_{A B C}$ exhibited by intermediate to high-albedo asteroids. We note again also the location of (21) Lutetia, that with an $\alpha_{\text {inv }}$ angle of $25^{\circ}$, lies in between Barbarians and 'normal' asteroids.

Figs 6 and 7 suggest a correlation between inversion angle and phase angle of $P_{\min }$ which is clearly shown in Fig. 8. Such a correlation, which may look straightforward, has not been explored much in the past. We note that this correlation is present for all but a few objects located at low values of $\alpha_{\text {inv }}$ and $\alpha\left(P_{\min }\right)$ (one of them being 


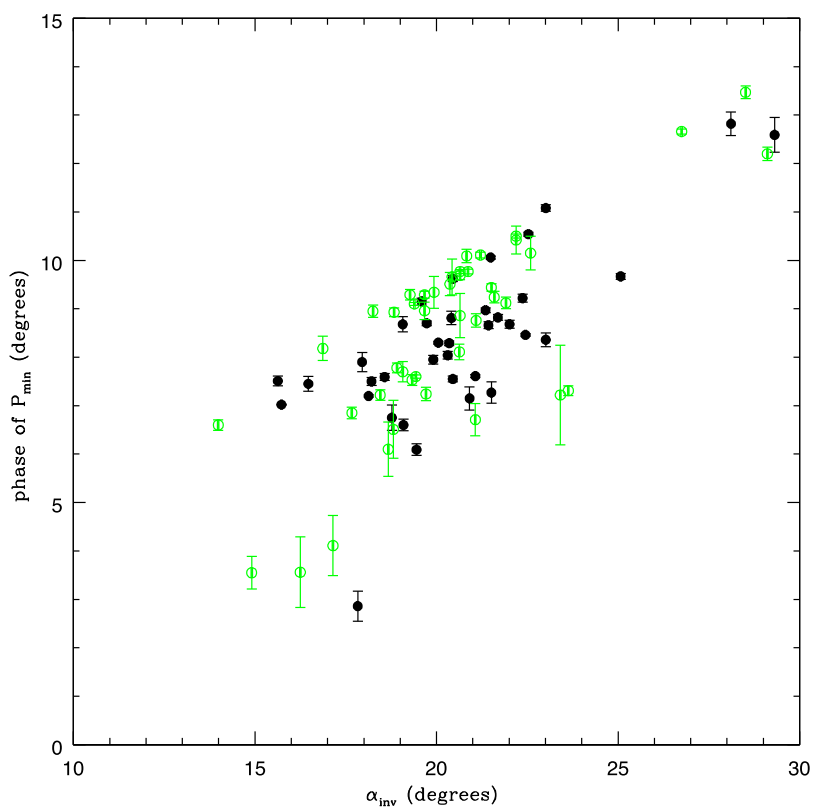

Figure 8. The $\alpha_{\text {inv }}$ inversion angle versus phase angle of $P_{\min }$ for the whole sample of asteroids considered in Table 1 and Paper I. Objects having fewer than 10 polarimetric measurements are indicated by open, green symbols.

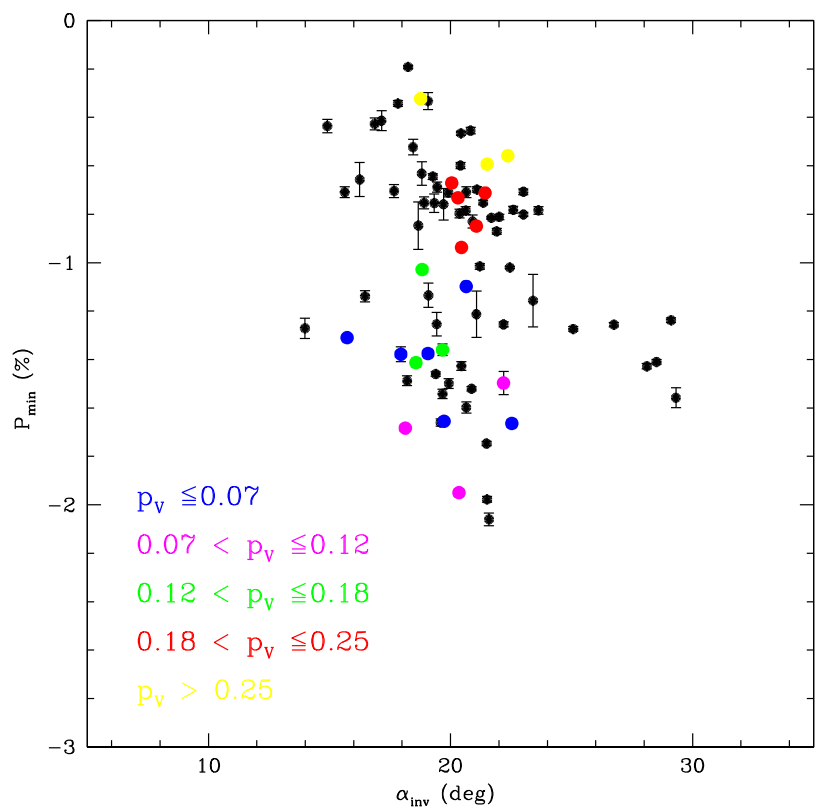

Figure 9. $P_{\min }$ versus inversion angle $\alpha_{\text {inv }}$ for the whole sample of asteroids listed in this paper and in Paper I. Objects having albedo values determined by S\&T (2006) are plotted in colour. Different colours correspond to different intervals of albedo, as specified in the figure.

(44) Nysa), and that the location of the Barbarians corresponds to the extrapolation of the linear trend exhibited by 'normal' asteroids.

The interpretation of the features discussed so far is not immediately obvious, but we believe this could be a useful input for current models of light scattering from rocky and/or icy planetary surfaces.

Finally, we show in Fig. 9 the relation between $\alpha_{\text {inv }}$ and $P_{\min }$. This is a classical relation analysed in the past by different authors to derive information on the likely properties of the particles which typically form the surface regolith of the asteroids (for a classical review, see Dollfus et al. 1989). Again, our analysis includes not only the objects listed in Table 1, but also the 22 asteroids included in the S\&T (2006) list that we considered in Paper I. In Fig. 9 the objects for which we have an albedo value given by S\&T (2006) are indicated by colour symbols, using different colours for different albedo classes.

Fig. 9 can be considered as an updated version of the analogous figure shown by Dollfus et al. (1989) in the Asteroids II book. We note that Dollfus et al. (1989) showed that asteroid data in the $P_{\min }-$ $\alpha_{\text {inv }}$ plane are found in a domain which is intermediate between one occupied by coarse rocks and one occupied by very thin lunar fines composed of particles smaller than $30 \mu \mathrm{m}$, according to laboratory measurements. We confirm that most asteroids of our sample occupy the region already found by Dollfus et al. (1989). Asteroids of increasingly higher albedo tend to occupy regions at the top of the asteroid domain, but there is some mixing at low-albedo values, with some objects having small S\&T (2006) albedos, below 0.07, which are found in this plot mixed with asteroids having albedos larger than 0.12. One of the mixed objects is (2) Pallas, with its albedo of 0.145 according to S\&T (2006), an unexpected value for an object belonging to the $B$ class, as discussed in Paper I.

The major difference with respect to the classical results by Dollfus et al. (1989), however, is the presence of some objects which are located well outside the typical domain of asteroids, which are found much closer to or within the domain found by Dollfus et al. (1989) for very pulverized material. These asteroids are Barbarians: (234) Barbara, (172) Baucis, (387) Aquitania, and (980) Anacostia, which all have inversion angles above $28^{\circ}$, and (236) Honoria, with an inversion angle above $26^{\circ}$. These objects occupy clearly anomalous locations in Fig. 9. Another object with a relatively high value of the inversion angle $\left(25^{\circ}\right)$ in Fig. 9 is (21) Lutetia. With a $P_{\min }$ value of about -1.27 , this asteroid would also be located in the domain of very pulverized rocks and lunar fines, according to Dollfus et al. (1989). Observations carried out from the ground and by the Rosetta probe during its fly-by of Lutetia have already provided evidence that this asteroid is unusual in several respects. It is encouraging, however, to mention that, according to Keihm et al. (2012), the thermal inertia of Lutetia is quite low, in very good agreement with the hypothesis that its surface could be rich in fine dust. Any further attempt of interpretation, however, must be postponed to future investigations.

\section{CONCLUSIONS AND FUTURE WORK}

The results of an extensive analysis of available asteroid polarimetric data, carried out in this paper and in Paper I, confirm that the study of the polarimetric properties of these objects is extremely interesting and a powerful tool for their physical characterization.

Several results shown in the previous sections deserve further studies mainly on the theoretical side, because we now have a wealth of information to test and extend current models of light-scattering phenomena. In particular, we find that some features of the negative polarization branch of phase-polarization curves (distributions of $\alpha_{\text {inv }}$ and $\alpha\left(P_{\min }\right)$ and the mutual relation between these parameters) are particularly interesting. The location of Barbarian asteroids in the $P_{\min }-\alpha_{\min }$ plane suggests that their surfaces are covered by extremely fine dust particles.

In this paper and Paper I, we give albedo values obtained from polarimetric parameters for a data set of 86 asteroids. This data set will hopefully increase rapidly in the years to come, as an effect of new campaigns of polarimetric observations. Some problems are certainly still open, including the apparently very high albedo of asteroid (44) Nysa. This is not atypical, as in general, high-albedo, 
$E$-class asteroids tend to display a rather large variation of albedo, depending on the choice of the polarimetric parameter adopted to obtain it. This implies that the calibration of the relation between geometric albedo and polarization parameters could yet see some further improvement.

We expect progress in the field to come in the near future from different directions. One possible development would be a systematic use of spectro-polarimetry. This will allow observers to profit from the results from spectroscopy and polarimetry, separately, plus the product of this merging of two separate techniques, namely the study of the variation of the linear polarization as a function of wavelength. Pioneering work in this respect has been already done by Belskaya et al. (2009) using broad-band polarimetric data obtained in different colours, while some of us have recently started a systematic campaign of spectro-polarimetric observations of asteroids at the ESO VLT and WHT (La Palma, Canary Islands) telescopes which has already provided encouraging results (Bagnulo, Cellino \& Sterzik 2015).

Finally, another essential input is provided by the in situ observations of the asteroid (4) Vesta performed by the Dawn spacecraft. Vesta is the only asteroid for which a clear variation of the degree of linear polarization as a function of rotation has been convincingly demonstrated (see Dollfus et al. 1989, and references therein). Some of us have recently carried out an extensive analysis of available 'polarimetric light curve' data of (4) Vesta, by computing the location of the sub-Earth point on Vesta at the epoch of different, ground-based polarimetric observations, in order to understand the relations with the varying, average properties of the surface seen by ground-based observers at different epochs, and to look for possible correlations with the albedo, topography and composition (Cellino et al. 2015b). In this study, we provided the first example of 'ground-truth' in asteroid polarimetry.

\section{ACKNOWLEDGEMENTS}

Partly based on observations carried out at the Complejo Astronómico El Leoncito, operated under agreement between the Consejo Nacional de Investigaciones Científicas y Técnicas de la República Argentina and the National Universities of La Plata, Córdoba, and San Juan. AC was partly supported by funds of the PRIN INAF 2011. AC and SB acknowledge support from COST Action MP1104 'Polarimetry as a tool to study the Solar system and beyond' through funding granted for Short Terms Scientific Missions and participations to meetings. RGH gratefully acknowledges financial support by CONICET through PIP 114-200801-00205. We thank the Referee, V. Rosenbush, for her careful review.

\section{REFERENCES}

Bagnulo S., Boehnhardt H., Muinonen K., Kolokolova L., Belskaya I., Barucci M. A., 2006, A\&A, 450, 1239

Bagnulo S., Belskaya I. N., Boehnhardt H., Kolokolova L., Muinonen K., Sterzik M., Tozzi G. P., 2011, J. Quant. Spectrosc. Radiat. Transfer, 112 , 2059
Bagnulo S., Cellino A., Sterzik M. F., 2015, MNRAS, 446, L11

Belskaya I. N. et al., 2005, Icarus, 178, 213

Belskaya I. N., Levasseur-Regourd A. C., Cellino A., Efimov Yu S., Shakhovskoy N. M., Hadamcik E., Bendjoya Ph., 2009, Icarus, 199, 97

Belskaya I. N., Bagnulo S., Barucci M. A., Muinonen K., Tozzi G. P., Fornasier S., Kolokolova L., 2010, Icarus, 210, 472

Bus S. J., Binzel R. P., 2002, Icarus, 158, 146

Cañada-Assandri M., Gil-Hutton R., Benavidez P., 2012, A\&A, 542, A11

Cellino A., Belskaya I. N., Bendjoya Ph., Di Martino M., Gil-Hutton R., Muinonen K., Tedesco E. F., 2006, Icarus, 180. 565

Cellino A., Bagnulo S., Tanga P., Novaković B., Delbò M., 2014, MNRAS, 439, L75

Cellino A., Bagnulo S., Gil-Hutton R., Tanga P., Cañada-Assandri M., Tedesco E. F., 2015a, MNRAS, 451, 3473 (Paper I)

Cellino A. et al., 2015b, MNRAS, submitted

Chamberlin A. B., McFadden L.-A., Schulz R., Schleicher D. G., Bus S. J., 1996, Icarus, 119, 173

Coradini A. et al., 2011, Science, 334, 492

Dollfus A., Wolff M., Geake J. E., Lupishko D. F., Dougherty L. M., 1989. in Binzel R. P., Gehrels T., Matthews M. S., eds, Asteroids II. Univ. Arizona Press, Tucson, AZ, p. 594

Gil-Hutton R., Cañada-Assandri M., 2011, A\&A, 529, A86

Gil-Hutton R., Cañada-Assandri M., 2012, A\&A, 539, A115

Gil-Hutton R., Mesa V., Cellino A., Bendjoya Ph., Peãloza L., Lovos F., 2008, A\&A, 482, 309

Gil-Hutton R., Cellino A., Bendjoya Ph., 2014, A\&A, 569, A122

Gradie J., Tedesco E. F., 1982, Science, 216, 1405

Keihm S. et al., 2012, Icarus, 221, 395

Masiero J. R., Cellino A., 2009, Icarus, 199, 333

Masiero J. R., Mainzer A. K., Grav T., Bauer J. M., Wright E. L., Mc Millan R. S., Tholen D. J., Blain A. W., 2012, ApJ, 749, A104

Muinonen K., Penttila A., Cellino A., Belskaya I. N., Delbo M., LevasseurRegourd A.-C., Tedesco E. F., 2009, Meteorit. Planet. Sci., 44, 1937

Rosenbush V. K., Kiselev N., Shevchenko V. G., Jockers K., Shakhovskoy N. M., Efimov Y. S., 2005, Icarus, 178, 222

Rosenbush V. K., Shevchenko V. G., Kiselev N., Sergeev A. V., Shakhovskoy N. M., Velichko F. P., Kolesnikov S. V., Karpov N. V., 2009, Icarus, 201, 655

Shevchenko V. G., Tedesco E. F., 2006. Icarus, 184, 211

Tedesco E. F., Williams J. G., Matson D. L., Veeder G. J., Gradie J. C., Lebofsky L. A., 1989, AJ, 97, 580

Tholen D. J., Barucci M. A., 1989, in Binzel R. P., Gehrels T., Matthews M. S., eds, Asteroids II. Univ. Arizona Press, Tucson, AZ, p. 298

This paper has been typeset from a $\mathrm{T}_{\mathrm{E}} \mathrm{X} / \mathrm{LAT}_{\mathrm{E}} \mathrm{X}$ file prepared by the author. 